\title{
Conformal radiotherapy of prostate cancer: What is the proper technique for sparing organs-at-risk?
}

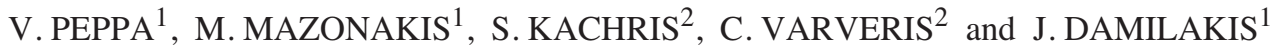 \\ ${ }^{1}$ Department of Medical Physics, Faculty of Medicine, University of Crete, Heraklion 710-03; ${ }^{2}$ Department \\ of Radiotherapy and Oncology, Heraklion University Hospital, Heraklion 711-10, Crete, Greece
}

Received October 6, 2009; Accepted October 30, 2009

DOI: $10.3892 /$ or_00000809

\begin{abstract}
The aim of the current study was to evaluate the optimal coplanar technique for conformal radiotherapy of prostate cancer. Twelve inoperable patients with prostatic carcinoma were examined. Five different techniques with three-, four- and six-fields were applied for treatment of prostate and seminal vesicles with or without lymph nodes. Treatment techniques were compared by using rectum, bladder and femoral heads dose-volume histogram data. A three-field arrangement consisting of an anterior and two lateral portals resulted in the maximum rectal sparing irrespectively of the irradiated area. The maximum femoral head sparing was achieved by the technique consisting of six oblique and lateral fields. The maximum bladder protection was observed with the box technique for treatment with large pelvic fields and with the arrangement consisting of two oblique and two lateral portals for conedown irradiation. In conclusion, the presented data allow the radiotherapists to select the proper irradiation technique associated with the maximum sparing of each organat-risk.
\end{abstract}

\section{Introduction}

Prostate cancer is the most common male malignant disease (1). Radiotherapy is often considered as the treatment of choice for the patients suffering from prostate cancer $(2,3)$. However, even with definitive treatment, it is estimated that $40 \%$ of men with clinically localized prostate cancer will experience biochemical relapse within 5 years (4). This high rate of biochemical failure reflects both the underestimation of patients with clinically localized disease who have occult pelvic nodal involvement, as well as the inadequate irradiation of these involved nodes. Surgical studies of pelvic

Correspondence to: Dr John Damilakis, Department of Medical Physics, Faculty of Medicine, University of Crete, P.O. Box 2208, Heraklion 710-03, Crete, Greece

E-mail: damilaki@med.uoc.gr

Key words: conformal radiotherapy, prostate cancer, lymph nodes, dose-volume histogram, treatment planning node dissection in prostate cancer patients have identified occult metastases in about $1 / 4$ of patients (5). Extended pelvic node dissections and prostate lymphography have demonstrated clinically occult lymph node involvement in pelvic nodes including the obturator, external iliac, internal iliac, common iliac, presacral and perirectal lymph nodes (5-7).

Conventional pelvic radiation fields based on bony landmarks are inadequate in covering the lymph nodes $(8,9)$. Magnetic resonance lymphangiograms have shown the inconsistent mapping of lymph nodes relative to bony landmarks and the presence of occult nodal metastases $(8,10)$. A large randomized study from the Radiation Therapy Oncology Group (RTOG 94-13) demonstrated a benefit in progressionfree survival in high-risk prostate cancer patients with the addition of whole-pelvis radiation over prostate radiation alone (11). This evidence suggests improved outcome with adequate lymph node coverage.

Three-dimensional treatment planning allows the radiotherapists and medical physicists to define the appropriate irradiation fields that cover all required lymph node areas. However, the above benefit of 3D conformal radiotherapy is counterbalanced by the exposure of the critical structures around the tumor to a relative high dose that may exceed the well-known dose tolerances. The selection of the proper irradiation technique is necessary in order to obtain the maximum sparing of healthy tissues and subsequently reduce possible side-effects. Several studies have compared a number of coplanar techniques employed for conformal prostate radiotherapy (18-22). However, none of the above studies has used treatment fields encompassing the lymph node area. The purpose of this study was to compare five different coplanar techniques for conformal radiotherapy of prostate plus seminal vesicles alone or with pelvic lymph nodes.

\section{Materials and methods}

CT examinations. Twelve inoperable patients with localized prostate cancer were enrolled in this study. The prostate, seminal vesicles and lymph nodes of the participants were radiographically normal. All patients had at least $15 \%$ risk of lymph node involvement, based on the criteria introduced by Roach et al $(12,13)$.

All patients underwent a pelvic CT examination on a 16detector-row CT scanner (Somatom Sensation 16, Siemens, Forcheim, Germany). To simulate treatment position, 

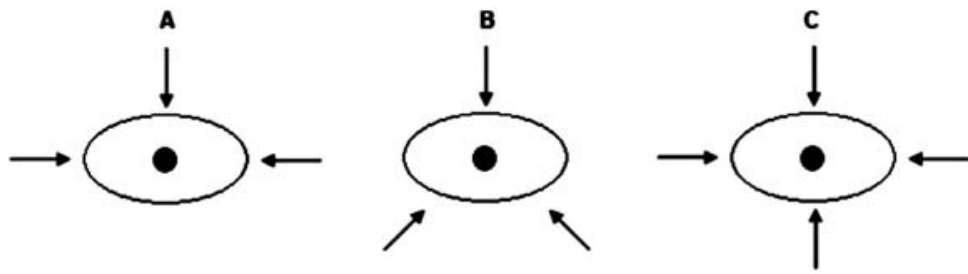

D

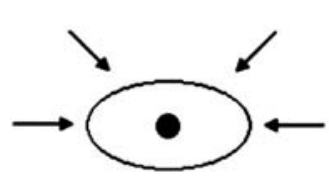

$\mathbf{E}$

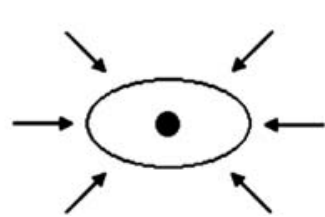

Figure 1. The five different techniques (A-E) considered in the two phases of irradiation. Lateral and oblique field irradiations were performed with or without wedges based on the requirement to achieve the best PTV coverage.

participants were scanned in supine position with their arms placed on the chest. Knee and ankle fixation cushion (Kneefix $^{\mathrm{TM}}$, Feetfix ${ }^{\mathrm{TM}}$, CIVCO Medical Solutions, USA) was used for patient immobilization. The CT scans covered the region from the L2/L3 vertebrae down to the level of the perineum, with section of $5 \mathrm{~mm}$ thickness and $5 \mathrm{~mm}$ intersection gap. All volumes-of-interest were outlined and approved by one attending physician. No attempts were made to account actively for the motion of these organs.

PTV and OARs delineation. CT data were transferred to a PC-based workstation having an image processing software (Focal, Version 4.3.3., CMS Inc., St. Louis, MO, USA). Organ contouring was performed by a radiotherapist experienced in prostate treatment. The following structures were delineated: prostate, seminal vesicles, pelvic lymph nodes, entire rectum and its contents, entire bladder and its contents and femoral heads. The rectal volume was contoured from the ischial tuberosity to the sigmoid flexure, whereas the entire femoral head to the lesser trochanter was included in the volume-of-interest. The pelvic nodes were contoured using the guidelines of Nutting et al (14) and Chao and Lin (15). Common iliac, external iliac, internal iliac, presacral, obturator and perirectal lymph nodes were included. The rectum, bladder and femoral heads were considered as the organs at risk (OARs) in this study.

The clinical target volume (CTV) consisted of prostate and seminal vesicles plus a uniform margin of $0.5 \mathrm{~cm}$. The addition of a non-uniform margin of $0.8 \mathrm{~cm}$ in the posterior side and of $1 \mathrm{~cm}$ in all other directions resulted in the planning target volume $\left(\mathrm{PTV}_{2}\right)$. The region encompassing the pelvic lymph nodes and $\mathrm{PTV}_{2}$ with a uniform margin of $1 \mathrm{~cm}$ corresponded to $\mathrm{PTV}_{1}$.

Conformal radiotherapy techniques. Three-dimensional conformal radiotherapy was administered in two phases. First, a total radiation dose of $5040 \mathrm{cGy}$ was delivered to $\mathrm{PTV}_{1}$. In the second conedown phase, a radiation dose of 1980 cGy was given to the region of $\mathrm{PTV}_{2}$. Radiotherapy was delivered with daily fractions of $180 \mathrm{cGy}$. Concurrent chemotherapy was also administered as part of an on-going protocol of our department (cisplatinum $20 \mathrm{mg} / \mathrm{m}^{2} / \mathrm{wk}$, docetaxel $12 \mathrm{mg} / \mathrm{m}^{2} / \mathrm{wk}$ ). External beam irradiation combined to concurrent chemotherapy has been successfully employed for the management of several malignant diseases (16-20). Reported experience has suggested that concurrent chemotherapy and radiotherapy up to tumor doses of $7380 \mathrm{cGy}$ is feasible for patients with locally advanced prostate cancer (21). In our department, a total radiation dose of $7020 \mathrm{cGy}$ is currently delivered to the tumor site.

The CT scans together with the outlines of all structures were subsequently transferred to our research 3D planning workstation (XiO 4.3.1, CMS Inc., St Louis, MO, USA). For each patient in the two treatment phases, the following isocentric coplanar plans were performed: i) plan A: a threefield arrangement consisting of an anterior field $\left(0^{\circ}\right)$ and a pair of left lateral/right lateral fields $\left(90^{\circ}\right.$ and $\left.270^{\circ}\right)$; ii) plan B: a three-field arrangement consisting of an anterior field $\left(0^{\circ}\right)$ and a pair of posterior oblique fields. The gantry angle for the left and right oblique field was $105^{\circ}-120^{\circ}$ and $240^{\circ}-255^{\circ}$, respectively. For each patient, the proper gantry angle was selected to obtain the optimum dose distribution; iii) plan $\mathrm{C}$ : a four-field arrangement consisting of opposed anterior/ posterior fields $\left(0^{\circ}\right.$ and $\left.180^{\circ}\right)$ and left lateral/right lateral fields $\left(90^{\circ}\right.$ and $\left.270^{\circ}\right)$; iv) plan D: a four-field arrangement consisting of left anterior oblique/right anterior oblique fields $\left(35^{\circ}\right.$ and $\left.225^{\circ}\right)$ and opposed left lateral/right lateral fields $\left(90^{\circ}\right.$ and $\left.270^{\circ}\right)$; v) plan E: a six-field arrangement of opposed left anterior oblique/right posterior oblique fields $\left(45^{\circ}\right.$ and $225^{\circ}$ ), opposed right anterior oblique/ left posterior oblique fields $\left(315^{\circ}\right.$ and $\left.135^{\circ}\right)$ and opposed left lateral/right lateral fields $\left(90^{\circ}\right.$ and $\left.270^{\circ}\right)$.

The afore-mentioned plans were selected as the most widely used irradiation techniques employed in every day clinical practice in our department. All patient treatments were designed to be performed with an $18 \mathrm{MV}$ photon beam produced by a linear accelerator (Primus, Siemens, Germany) equipped with multileaf collimator (MLC). Four filters with wedge angles of $15^{\circ}, 30^{\circ}, 45^{\circ}$ and $60^{\circ}$ are available in the linear accelerator of our department. The irradiation techniques are graphically shown in Fig. 1.

Concerning the three field techniques, the beam weights for the anterior and the pair of lateral or oblique fields were set at $0.4,0.3$ and 0.3 , respectively. Equally weighted field 
irradiations were assumed for the plans C, D and E. The above selected weights can result in a homogenous dose distribution inside the PTV of both treatment phases. A 6-mm margin around the $\mathrm{PTV}_{1}$ and $\mathrm{PTV}_{2}$ was used to account for beam penumbra, ensuring $95 \%$ prescription dose coverage to the periphery of the irradiated areas. Lateral and oblique field irradiations were generated using wedge filters, only when an improvement on the dose homogeneity within the $\mathrm{PTV}_{1}$ and/or $\mathrm{PTV}_{2}$ was observed. For each treatment plan, the wedge angles were selected from a medical physisist, in respect to the best coverage of $\mathrm{PTV}_{1}$ and $\mathrm{PTV}_{2}$ and the maximal sparing of the OARs. In all treatment plans the isodose lines were normalized to the field isocentre.

Dose-volume histograms. Dose-volume histograms (DVHs) were calculated for $\mathrm{PTV}_{1}, \mathrm{PTV}_{2}$, rectum, bladder and femoral heads using the treatment planning system. The optimum dose distribution for each plan was evaluated considering the data from the DVH and the coverage of the treatment volume as presented by isodose lines. The percentage of the volume of interest receiving a radiation dose equal to $\mathrm{x} \%$ of the prescribed isocentric dose was denoted $\mathrm{V}_{\mathrm{x}}$. The $\mathrm{V}_{\mathrm{x}}$ of each OAR was determined using the DVH data.

The comparative evaluation of the five different irradiation techniques was performed using the mean $\mathrm{V}_{\mathrm{x}}$ values for each OAR. For the rectum and the bladder, $V_{80}$ and $V_{90}$ values were determined, whereas the $\mathrm{V}_{50}$ value was obtained for the femoral heads. The femoral head DVH data were averaged over the left and right sides for every patient and a mean $\mathrm{V}_{50}$ value was calculated.

The calculated $V_{x}$ values of each technique were compared with the respective values associated with the other irradiation techniques. Multiple comparisons were performed through a Wilcoxon matched pairs test, using the software Prism, version 4.00 (GraphPadPrism software Inc., CA, USA). A P-value of $<0.05$ was considered to indicate statistical significance. The null hypothesis was that there was no difference between the techniques.

\section{Results}

First treatment phase. In the first phase of irradiation, giving 5040 cGy to the treatment volume, the $\mathrm{V}_{95}$ value for the PTV varied from 92.1 to $97.3 \%$. The minimum $\mathrm{V}_{95}$ value recorded for the gross tumor volume $\left(\mathrm{GTV}_{1}\right)$ was $96.3 \%$ for all patients and all the considered techniques.

The mean rectal $\mathrm{V}_{80}$ and $\mathrm{V}_{90}$ values in respect to the applied treatment technique are shown in Fig. 2A. The lowest rectal $\mathrm{V}_{80}$ and $\mathrm{V}_{90}$ values were recorded in plan $\mathrm{A}$. The highest rectal $\mathrm{V}_{80}$ and $\mathrm{V}_{90}$ values were marked in plans $\mathrm{E}$ and $\mathrm{B}$, respectively. Technique $A$ had a significantly lower $V_{80}$ value than any of the other plans $(\mathrm{P}=0.0005)$. Considering $\mathrm{V}_{90}$, technique $\mathrm{A}$ was significantly different from techniques $\mathrm{B}$ $(\mathrm{P}=0.0005), \mathrm{C}(\mathrm{P}=0.0010), \mathrm{D}(\mathrm{P}=0.0015)$ and $\mathrm{E}(\mathrm{P}=0.0005)$. The mean rectal dose associated with technique $\mathrm{A}$ was $4236 \pm 325$ cGy.

The mean bladder $\mathrm{V}_{80}$ and $\mathrm{V}_{90}$ values are shown in Fig. 2B. It can be observed that plan $\mathrm{C}$ had the lowest bladder $\mathrm{V}_{80}-\mathrm{V}_{90}$
A

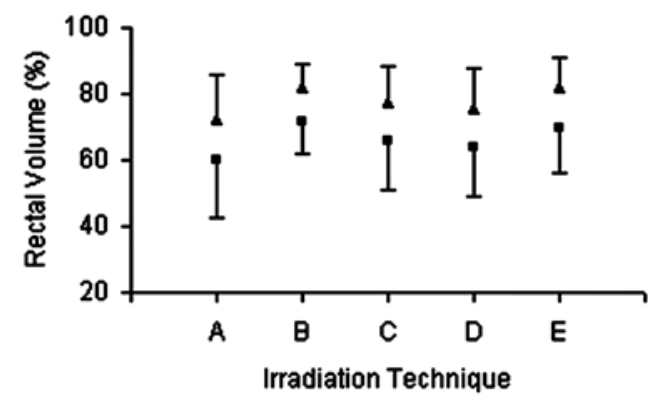

\begin{tabular}{l} 
- V80 \\
- V90 \\
\hline
\end{tabular}

B

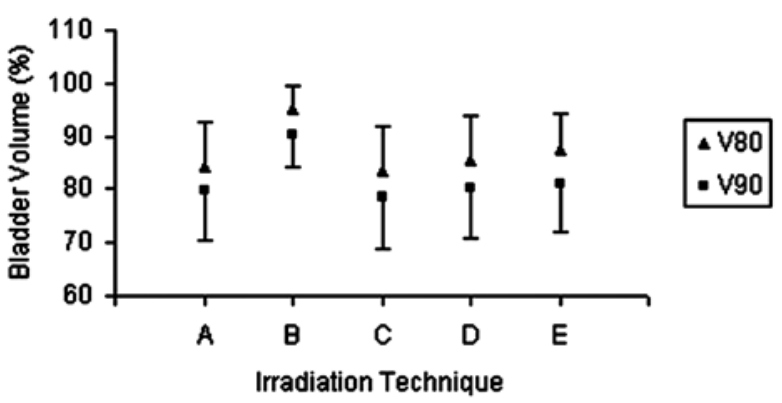

C

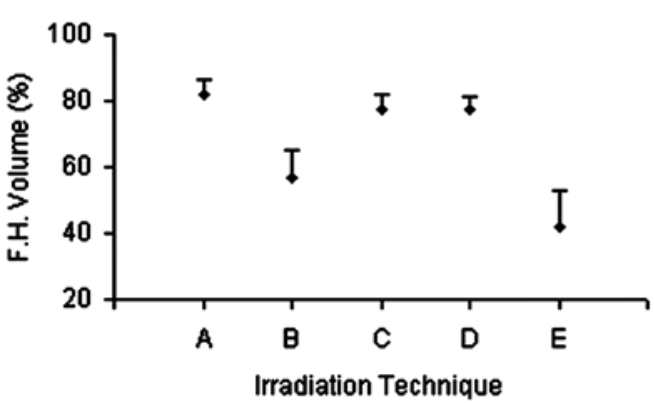

$\bullet$ V50

Figure 2. The mean irradiated (A) rectal volumes $\left(\mathrm{V}_{80}, \mathrm{~V}_{90}\right)$, (B) bladder volumes $\left(\mathrm{V}_{80}, \mathrm{~V}_{90}\right)$ and $(\mathrm{C})$ femoral head (F.H.) volume $\left(\mathrm{V}_{50}\right)$ as a function of the plan type in the first treatment phase. Error bars are one standard deviation and they are shown in one direction.

values, whilst plan $\mathrm{B}$ resulted in the highest bladder $\mathrm{V}_{80}-\mathrm{V}_{90}$ values. When considering $\mathrm{V}_{80}$, technique $\mathrm{C}$ was significantly different from techniques $\mathrm{B}(\mathrm{P}=0.0010), \mathrm{E}(\mathrm{P}=0.0005)$ and $\mathrm{D}$ $(\mathrm{P}=0.0068)$. In terms of $\mathrm{V}_{90}$, technique $\mathrm{C}$ was significantly different from techniques $\mathrm{B}(\mathrm{P}=0.0010)$ and $\mathrm{E}(\mathrm{P}=0.0005)$. No significant difference was found between plans $\mathrm{A}$ and $\mathrm{C}$ ( $\mathrm{P}>0.05$ ). However, it should be mentioned that technique $\mathrm{C}$ resulted in a lower mean bladder dose of $4687 \pm 177 \mathrm{cGy}$ than the dose of $4758 \pm 176 \mathrm{cGy}$ from technique A.

The mean femoral head $V_{50}$ values are summarized in Fig. 2C. It is clear that the lowest $\mathrm{V}_{50}$ value appeared with plan $\mathrm{E}$, while plan $\mathrm{A}$ demonstrated the highest $\mathrm{V}_{50}$ value. The resultant $\mathrm{V}_{50}$ values from all the other techniques were significantly higher than that from plan $\mathrm{E}(\mathrm{P}<0.05)$. The mean femoral head dose recorded from technique $\mathrm{E}$ was 2608 149 cGy. 
A

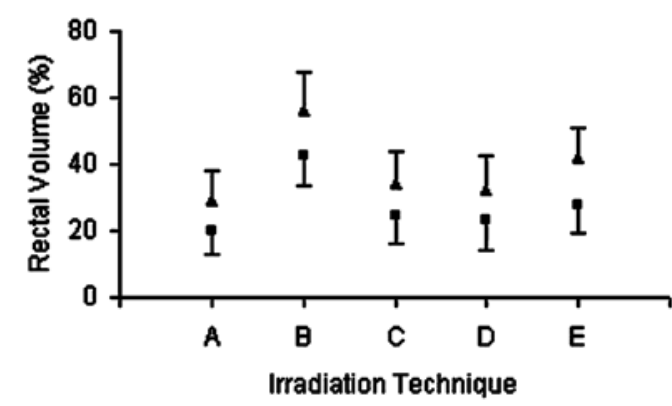

B

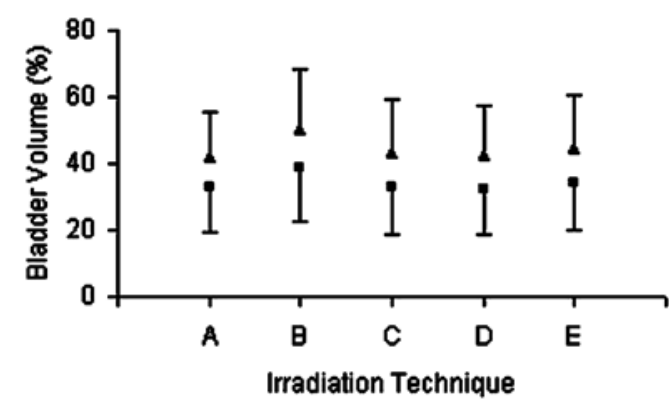

C

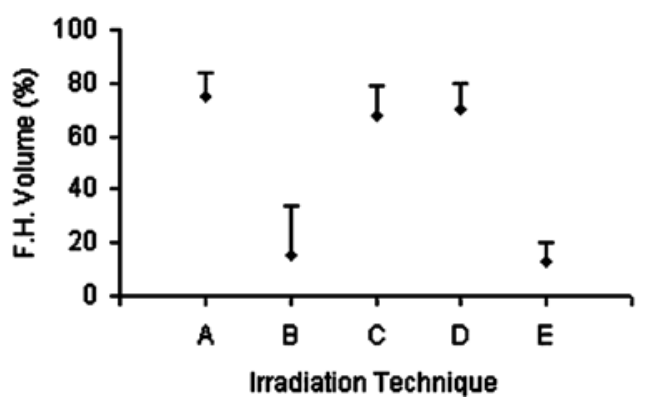

Figure 3. The mean irradiated (A) rectal volumes $\left(\mathrm{V}_{80}, \mathrm{~V}_{90}\right)$, (B) bladder volumes $\left(\mathrm{V}_{80}, \mathrm{~V}_{90}\right)$ and $(\mathrm{C})$ femoral head (F.H.) volume $\left(\mathrm{V}_{50}\right)$ as a function of the plan type in the second treatment phase. Error bars are one standard deviation and they are shown in one direction.

Second treatment phase. In the second phase of irradiation, delivering $1980 \mathrm{cGy}$ to the irradiated area, it was marked that the $\mathrm{V}_{95}$ value for the $\mathrm{PTV}_{2}$ varied from 92.1 to $97.1 \%$. In terms of $\mathrm{GTV}_{2}$, the minimum $\mathrm{V}_{95}$ value recorded was $98.7 \%$ for all the treatment techniques.

The mean irradiated rectal $V_{80}$ and $V_{90}$ volumes are shown in Fig. 3A. Similar to the first phase, the lowest rectal $V_{80}$ and $V_{90}$ values were recorded for plan $A$. The highest $V_{80}$ and $\mathrm{V}_{90}$ values were observed in plan $\mathrm{B}$. Technique $\mathrm{A}$ irradiated a significantly smaller volume of rectum than techniques B, C, $\mathrm{D}$ and $\mathrm{E}\left(\mathrm{V}_{80}, \mathrm{P}=0.0005 ; \mathrm{V}_{90}, \mathrm{P} \leq 0.0010\right)$. The resultant mean rectal dose from technique $\mathrm{A}$ was $1188 \pm 159 \mathrm{cGy}$.

The mean bladder $V_{80}$ and $V_{90}$ values are summarized in Fig. 3B. The lowest $V_{80}$ value was observed for treatment plan $A$. The $\mathrm{V}_{80}$ value obtained from patient irradiation with technique A was significantly lower than that with technique $B(P=0.0010)$, whereas no significant differences were observed between treatment plan $\mathrm{A}$ and plans $\mathrm{C}, \mathrm{D}$ and $\mathrm{E}$ $(\mathrm{P}>0.05)$. When considering $\mathrm{V}_{90}$, technique $\mathrm{D}$ had lower value than the other examined strategies. Compared to plan $\mathrm{D}$, statistically significant difference was recorded only between techniques $\mathrm{D}$ and $\mathrm{B}(\mathrm{P}=0.0068)$. It should be noted that treatment techniques $\mathrm{A}$ and $\mathrm{D}$ resulted in almost the same bladder $\mathrm{V}_{80}$ value (Fig. 3B), while the mean bladder dose from plan $\mathrm{D}$ was lower. The mean bladder doses from techniques $\mathrm{A}$ and $\mathrm{D}$ were $1340 \pm 287$ and $1296 \pm 285 \mathrm{cGy}$, respectively.

The mean femoral head $\mathrm{V}_{50}$ values are demonstrated in Fig. 3C. The lowest $V_{50}$ value was recorded in plan $E$. The mean $\mathrm{V}_{50}$ value from plan $\mathrm{E}$ was significantly lower than those from plans $\mathrm{A}, \mathrm{C}$ and $\mathrm{D}(\mathrm{P}=0.0005)$, whereas it was comparable to that from plan $\mathrm{B}(\mathrm{P}=0.97)$. It should be reported that the mean femoral head dose from technique $\mathrm{E}$ was $784 \pm 63 \mathrm{cGy}$.

\section{Discussion}

In the current study we compared five different techniques for conformal irradiation of prostate and seminal vesicles or prostate, seminal vesicles and lymph nodes. This comparison was based on the calculated dose-volume histograms of the rectum, bladder and femoral heads. The rectum is the main dose-limiting organ for pelvic irradiation in prostate cancer (22). The results of our investigation indicated that for both treatment phases, patient irradiation with a three-field arrangement consisting of an anterior portal and a pair of lateral fields provided the maximum rectal sparing. This may be attributed to the absence of a posterior portal that may directly expose the rectum to primary irradiation.

Regarding the urinary bladder, the box irradiation technique resulted in the lowest $\mathrm{V}_{80}$ and $\mathrm{V}_{90}$ values, during the first treatment phase giving $5040 \mathrm{cGy}$ to the tumor site. For the conedown irradiation, the arrangement consisting of two oblique and two lateral fields seem to be the preferred approach for bladder sparing. Our data showed that different irradiation techniques can result in the maximum bladder sparing in each treatment phase. This might be attributed to the different size of PTV and, therefore, to the different part of urinary bladder included in the irradiated area from phase to phase.

The six-field arrangement resulted in the maximum sparing of femoral heads irrespective of the pelvic field size applied to prostate cancer patients. For the conedown irradiation, technique $\mathrm{B}$ consisting of an anterior and a pair of posterior oblique fields resulted in almost the same $V_{80}$ and $\mathrm{V}_{90}$ values with those from the six-field treatment technique. In the second treatment phase, technique B can also be employed for the protection of femoral heads taking into account the high time cost related to the six-field treatment in every day clinical practice.

To the best of our knowledge, there is no reported experience on the optimum technique for irradiating a region encompassing prostate, seminal vesicles and lymph nodes. Our results can be directly compared only with those obtained with a CTV including prostate and seminal vesicles. 
The results of our study are in agreement with those presented by most of the studies dealing with conformal conedown irradiation therapy of prostate cancer. Neal et al (23) found that a great femoral head sparing was demonstrated with the six-field technique, whereas box technique provided a better sparing of the rectum in respect to the other examined strategies. In a study reported by Fiorino et al (24), it was concluded that the best sparing of the rectum was achieved with the three-field technique consisting of an anterior and a pair of lateral portals. Moreover, there was a benefit for the femoral heads with the six-field technique and the three-field arrangement consisting of an anterior and a pair of posterior oblique fields. Khoo et al (25) and Hille et al (26) showed a better sparing of the rectum with the three-field technique with two lateral fields. Hille et al (26) also reported that the forenamed technique was comparable to box technique, in terms of bladder. These results are congruent with the results of our study.

Regarding the bladder sparing, Fiorino et al (24) proposed the use of a three-field technique consisting of an anterior field and a pair of posterior oblique portals. However, our dosimetric data showed that different treatment strategies can provide the maximum bladder sparing. Bedford et al (27) only compared a variety of four-field treatment plans. In terms of rectal sparing, they found that the arrangement consisting of two lateral and two anterior oblique fields was superior to the box technique. Our results showed that the box technique provides a better rectal sparing than the four-field arrangement consisting of two oblique and two lateral portals.

This study has several limitations. A relatively small number of inoperable prostate cancer patients were examined. Moreover, the obtained dosimetric data refer to patients treated in prone position. Special consideration should be given whenever the presented dosimetric results are applied in routine clinical practice. The DVHs cannot take into account the daily variations in filling rectum and urinary bladder that may be presented during the entire treatment course $(28,29)$.

In conclusion, the selection of the proper irradiation technique during conformal radiotherapy of prostate cancer is strongly related to the irradiated area, the tumor dose and the dose tolerance of critical organs surrounding the treatment volume. Our results clearly revealed that there is no single irradiation strategy providing simultaneously the maximum sparing of all OARs in each treatment phase. The dosimetric data presented in this study may be of value for radiotherapists and medical physicists whenever difficult decisions about the optimum sparing of rectum, urinary bladder and femoral heads should be made.

\section{References}

1. Jemal A, Siegel R, Ward E, Hao Y, Xu J, Murray T and Thun MJ: Cancer statistics, 2008. CA Cancer J Clin 58: 71-96, 2008.

2. Koukourakis MI and Touloupidis S: External beam radiotherapy for prostate cancer: current position and trends. Anticancer Res 26: 485-494, 2006

3. Ogawa K, Nakamura K, Sasaki T, Onishi H, Koizumi M, Shioyama Y, Araya M, Mukumoto N, Mitsumori M and Teshima T; Japanese Patterns of Care Study Working Subgroup of Prostate Cancer: External beam radiotherapy for clinically localized hormone-refractory prostate cancer: clinical significance of Nadir prostate-specific antigen value within 12 months. Int J Radiat Oncol Biol Phys 74: 759-765, 2009.
4. Kestin LL, Vicini FA, Ziaja EL, Stromberg JS, Frazier RC and Martinez AA: Defining biochemical cure for prostate carcinoma patients treated with external beam radiation therapy. Cancer 86: 1557-1566, 1999.

5. Heidenreich A, Ohlmann $\mathrm{CH}$ and Polyakov S: Extended pelvic lymphadenectomy in patients undergoing radical prostatectomy: high incidence of lymph node metastasis. J Urol 167: 1681-1686, 2002.

6. Allaf ME, Palapattu GS, Trock BJ, Carter HB and Walsh PC: Anatomical extent of lymph node dissection: impact on men with clinically localized prostate cancer. J Urol 172: 1840-1844, 2004.

7. Takashima H, Egawa M, Imao T, Fukuda M, Yokoyama K and Namiki M: Validity of sentinel lymph node concept for patients with prostate cancer. J Urol 171: 2268-2271, 2004.

8. Shih HA, Harisinghani M, Zietman AL, Wolfgang JA, Saksena M and Weissleder R: Mapping of nodal disease in locally advanced prostate cancer: rethinking the clinical target volume for pelvic nodal irradiation based on vascular rather than bony anatomy. Int J Radiat Oncol Biol Phys 63: 1262-1269, 2005.

9. Taylor A, Rockall AG, Reznek RH and Powell ME: Mapping pelvic lymph nodes: guidelines for delineation in intensitymodulated radiotherapy. Int J Radiat Oncol Biol Phys 63: 1604-1612, 2005.

10. Dinniwell R, Chan P, Czarnota G, Haider MA, Jhaveri K, Jewett M, Fyles A, Jaffray D and Milosevic M: Pelvic lymph node topography for radiotherapy treatment planning from ferumoxtran-10 contrast-enhanced magnetic resonance imaging. Int J Radiat Oncol Biol Phys 74: 844-851, 2009.

11. Roach M III, DeSilvio M, Lawton C, Uhl V, Machtay M, Seider MJ, Rotman M, Jones C, Asbell SO, Valicenti RK, Han S, Thomas CR Jr and Shipley WS; Radiation Therapy Oncology Group 9413: Phase III trial comparing whole-pelvic versus prostate-only radiotherapy and neoadjuvant versus adjuvant combined androgen suppression: Radiation Therapy Oncology Group 9413. J Clin Oncol 21: 1904-1911, 2003.

12. Roach M III, Marquez C, Yuo HS, Narayan P, Coleman L, Nseyo UO, Navvab Z and Carroll PR: Predicting the risk of lymph node involvement using the pre-treatment prostate specific antigen and Gleason score in men with clinically localized prostate cancer. Int J Radiat Oncol Biol Phys 28: 33-37, 1994.

13. Roach M III, Chen A, Song J, Diaz A, Presti J Jr and Carroll P: Pretreatment prostate-specific antigen and Gleason score predict the risk of extracapsular extension and the risk of failure following radiotherapy in patients with clinically localized prostate cancer. Semin Urol Oncol 18: 108-114, 2000.

14. Nutting CM, Convery DJ, Cosgrove VP, Rowbottom C Padhani AR, Webb S and Dearnaley DP: Reduction of small and large bowel irradiation using an optimized intensitymodulated pelvic radiotherapy technique in patients with prostate cancer. Int J Radiat Oncol Biol Phys 48: 649-656, 2000.

15. Chao KS and Lin M: Lymphangiogram-assisted lymph node target delineation for patients with gynecologic malignancies. Int J Radiat Oncol Biol Phys 54: 1147-1152, 2002.

16. Kogashiwa Y, Yamauchi K, Nagafuji H, Matsuda T, Tsubosaka T, Tsutsumi T, Karaho T and Kohno N: Concurrent chemoradiotherapy for organ function preservation in advanced patients with hypopharyngeal and laryngeal cancer. Oncol Rep 22: 1163-1167, 2009.

17. Kusumoto S, Hirose T, Fukayama M, Kataoka D, Hamada K, Sugiyama T, Shirai T, Yamaoka T, Okuda K, Ohnishi T, Ohmori T, Kadokura M and Adachi M: Induction chemoradiotherapy followed by surgery for locally advanced nonsmall cell lung cancer. Oncol Rep 22: 1157-1162, 2009.

18. Yang SH, Hong YK, Yoon SC, Kim BS, Lee YS, Lee TK, Lee KS, Jeun SS, Kim MC and Park CK: Radiotherapy plus concurrent and adjuvant procarbazine, lomustine and vincristine chemotherapy for patients with malignant glioma. Oncol Rep 17: 1359-1364, 2007

19. Yokoyama Y, Takano T, Nakahara K, Shoji T, Sato H, Yamada H, Yaegashi N, Okamura K, Kurachi H, Sugiyama T, Tanaka T, Sato A, Tase T and Mizunuma H: A phase II multicenter trial of concurrent chemoradiotherapy with weekly nedaplatin in advanced uterine cervical carcinoma: Tohoku Gynecologic Cancer Unit Study. Oncol Rep 19: 1551-1556, 2008.

20. Varveris H, Petinelli E, Stratakis J and Mazonakis M: Phase I study of weekly topotecan combined to concurrent external cranial irradiation in adults with glioblastoma multiforme of the brain. Oncol Rep 19: 447-455, 2008. 
21. Sanfilippo NJ, Taneja SS, Chachoua A, Lepor H and Formenti SC: Phase I/II study of biweekly paclitaxel and radiation in androgen-ablated locally advanced prostate cancer. J Clin Oncol 26: 2973-2978, 2008.

22. Dearnaley DP, Khoo VS, Norman AR, Meyer L, Nahum A, Tait D, Yarnold J and Horwich A: Comparison of radiation side-effects of conformal and conventional radiotherapy in prostate cancer: a randomised trial. Lancet 353: 267-272, 1999.

23. Neal AJ, Oldham M and Dearnaley DP: Comparison of treatment techniques for conformal radiotherapy of the prostate using dose-volume histograms and normal tissue complication probabilities. Radiother Oncol 37: 29-34, 1995.

24. Fiorino C, Reni M, Cattaneo GM, Bolognesi A and Calandrino R Comparing 3-, 4- and 6-field techniques for conformal irradiation of prostate and seminal vesicles using dose-volume histograms. Radiother Oncol 44: 251-257, 1997.

25. Khoo VS, Bedford JL, Webb S and Dearnaley DP: An evaluation of three-field coplanar plans for conformal radiotherapy of prostate cancer. Radiother Oncol 55: 31-40, 2000.
26. Hille A, Töws N and Hess CF: A comparison of three-field and four-field techniques in different clinical target volumes in prostate cancer irradiation using dose volume histograms: a prospective three-dimensional analysis. Br J Radiol 79: 148-157, 2006.

27. Bedford JL, Khoo VS, Oldham M, Dearnaley DP and Webb S: A comparison of coplanar four-field techniques for conformal radiotherapy of the prostate. Radiother Oncol 51: 225-235, 1999.

28. Stasi M, Munoz F, Fiorino C, Pasquino M, Baiotto B, Marini P, Malinverni G, Valdagni R and Gabriele P: Emptying the rectum before treatment delivery limits the variations of rectal dosevolume parameters during 3DCRT of prostate cancer. Radiother Oncol 80: 363-370, 2006.

29. De Crevoisier R, Melancon AD, Kuban DA, Lee AK, Cheung RM, Tucker SL, Kudchadker RJ, Newhauser WD, Zhang L, Mohan R and Dong L: Changes in the pelvic anatomy after an IMRT treatment fraction of prostate cancer. Int J Radiat Oncol Biol Phys 68: 1529-1536, 2007 